\title{
Distancia entre plantas y dosis de nitrógeno y potasio en sandía sin semillas fertirrigada
}

\author{
Anderson Luiz Feltrim ${ }^{(1)}$, Arthur Bernardes Cecílio Filho(1), Marcus Vinicius Gonsalves ${ }^{(1)}$, Luiz Carlos Pavani(1), \\ José Carlos Barbosa ${ }^{(1)}$ y Juan Waldir Mendoza Cortez ${ }^{(1)}$
}

(1)Universidade Estadual Paulista, Faculdade de Ciências Agrárias e Veterinárias, Via de Acesso Prof. Paulo Donato Castellane, s/no, CEP 14884-900 Jaboticabal, SP. E-mail: andluizfel@yahoo.com.br, rutra@fcav.unesp.br, marcusfcav@yahoo.com.br, Icpavani@fcav.unes.br, jcbarbosa@fcav.unesp.br, invic64@hotmail.com

Resumen - El objetivo de este trabajo fue evaluar los efectos de dosis de $\mathrm{N} \mathrm{y}_{2} \mathrm{O}$, vía fertirrigación por goteo, y de la distancia entre plantas sobre la productividad del híbrido Shadow de sandía sin semillas. El experimento se realizó de agosto a octubre de 2008 en el Município de Tupã, SP, Brazil. Se avaluaran cuatro dosis de $\mathrm{N}+\mathrm{K}_{2} \mathrm{O}\left(79,8+106,7,106,4+142,2,133,0+177,7\right.$, y $159,6+213,2 \mathrm{~kg} \mathrm{ha}^{-1}$, respectivamente), y cuatro distancias entre plantas $(0,5,1,0,1,5$ y 2,0 m), en hileras espaciadas de 2,0 m. Se utilizó el diseño experimental de bloques al azar, en parcelas subdivididas, con tres repeticiones. Las variables evaluadas fueron: concentración foliar de $\mathrm{N}$ y K, peso medio de frutos, número de frutos y productividad total y comercial; y número de frutos en las clases de 3 a 5,5 a 7,7 a $9 \mathrm{~kg}$ y con más de $9 \mathrm{~kg}$, por planta y por hectárea. Hubo efecto del factor dosis de $\mathrm{N}+\mathrm{K}_{2} \mathrm{O}$ sobre la concentración foliar de $\mathrm{K}$, y la productividad total y comercial de la sandía híbrido Shadow disminuyó linealmente con el aumento de la distancia entre plantas. Sin embargo, no hubo efecto significativo de la interacción ni efecto individual de los factores sobre las otras variables avaliadas.

Términos para indexación: Citrullus lanatus, nitrógeno, nutrición mineral, población de plantas.

\section{Spacing between plants and doses of nitrogen and potassium in fertigated seedless watermelon}

\begin{abstract}
The objective of this work was to evaluate the effects of $\mathrm{N}$ and $\mathrm{K}_{2} \mathrm{O}$ doses, applied by a drip fertigation system, and of distances between plants on the productivity of the Shadow hybrid of seedless watermelon. The experiment was carried out from August to October 2008, at Tupã, SP, Brazil. Four doses of $\mathrm{N}+\mathrm{K}_{2} \mathrm{O}\left(79.8+106.7,106.4+142.2,133.0+177.7\right.$, and $\left.159.6+213.2 \mathrm{~kg} \mathrm{ha}^{-1}\right)$ and four spacings between plants $(0.5,1.0,1.5$, and $2.0 \mathrm{~m})$ were evaluated in $2.0-\mathrm{m}$ spaced rows. A randomized complete block design with split plots and three replicates was used. Evaluated variables were: $\mathrm{N}$ and $\mathrm{K}$ foliar content, mean fruit weight, number of fruit and total and commercial productivity; and number of fruit from classes 3 to $5 \mathrm{~kg}, 5$ to $7 \mathrm{~kg}, 7$ to $9 \mathrm{~kg}$, and above $9 \mathrm{~kg}$ per plant and per hectare. Nitrogen and potassium doses significantly affected K foliar content, and total and commercial productivity of the hybrid Shadow decreased linearly with the increase in spacing between plants. However, there was no effect of the interaction between factors or from the factors themselves on the other evaluated variables.
\end{abstract}

Index terms: Citrullus lanatus, nitrogen, mineral nutrition, plant population.

\section{Introducción}

La sandía es cultivada prácticamente en todo el Brasil. De 1999 a 2006, la producción anual promedio fue de 1,83 millones de toneladas, en 81.892 ha. Los mayores estados productores fueron Rio Grande do Sul (428 mil toneladas), Bahía (244 mil toneladas) y São Paulo (207 mil toneladas) (Instituto FNP, 2009).

La producción y la calidad de la sandía están relacionadas con factores genéticos, climáticos y fitotécnicos, en que la nutrición mineral és de importancia fundamental. Una tecnología muy utilizada en este cultivo es la fertirrigación, pero en
Brasil su aplicación es aún incipiente, y el principal problema es su manejo incorrecto, en razón de la falta de informaciones técnicas disponibles y de su utilización empírica (Villas Bôas et al., 2001).

En la fertilización convencional, sólo un tercio de los fertilizantes nitrogenados y potásicos son utilizados por las plantas (Alfaia, 1997), mientras que con la aplicación de fertirrigación hay un aumento de la eficiencia de la fertilización (Andrade Júnior et al., 2006b). En el Estado de Ceará, con un buen manejo de la fertirrigación, la producción de sandía está entre 50 a $70 \mathrm{Mg} \mathrm{ha}^{-1}$, mientras que su productividad promedio es de $35 \mathrm{Mg} \mathrm{ha}^{-1}$. 
La cantidad de nutrientes requeridos por la sandía depende principalmente de la forma de aplicación, de la población de plantas, de la cultivar utilizada y de la región productora. Nitrógeno y potasio son los nutrientes más extraídos por este cultivo (Grangeiro \& Cecílio Filho, 2004, 2005). En el Estado de São Paulo, la fertilización convencional de la sandía es realizada en forma fraccionada, aplicándose de 50 a $100 \mathrm{~kg} \mathrm{ha}^{-1}$ de $\mathrm{N}$ y de $\mathrm{K}_{2} \mathrm{O}$ a la siembra y a los 15, 30 y 50 días después de la emergencia, (Trani et al., 1997). Andrade Júnior et al. (2006a) emplearon fertirrigación, con dosis de $\mathrm{N}$ a $0,40,80,120$ y $160 \mathrm{~kg} \mathrm{ha}^{-1}$, y verificaron que $97,6 \mathrm{~kg} \mathrm{ha}^{-1}$ de $\mathrm{N}$ optimizó la productividad comercial; tambiém con fertirrigación. Por otro lado, Morais et al. (2008) verificaron máxima productividad de sandia (267 kg ha-1), aplicando dosis de 75 a $300 \mathrm{~kg} \mathrm{ha}^{-1}$ de N y riego de $421 \mathrm{~mm}$.

En cucurbitaceas, altas densidades de plantación producen un gran número de frutos por área, pero con el tamaño, peso y número de frutos por planta reducidos, debido principalmente a la competencia entre ellas (Robinson \& Walters, 1997); ya en menores densidades, se ha verificado lo contrario. Para sandía, se recomiendan distanciamientos de 2,0 a 3,0 $\mathrm{m}$ x 1,0 a $1,5 \mathrm{~m}$ (Filgueira, 2008) y 2,5 a 3,0 m x 1,5 a 2,0 m (Trani et al., 1997) entre hileras y plantas, respectivamente. Pero la elección depende de la cultivar utilizada; sin embargo, estas distancias son empleadas en cultivares con semillas, que generalmente tienen mayor crecimiento vegetativo y mayor tamaño de frutos, en comparación con las cultivares sin semillas.

Para aumentar la productividad en los cultivos, se requiere una revisión constante de la fertilización en relación a la densidad poblacional, debido a la comercialización de nuevas cultivares, surgimiento de nuevas regiones de cultivo y de las características de mercado de la hortaliza. Las variaciones en el distanciamiento entre plantas o entre hileras pueden causar cambios morfológicos que alteran el desarrollo de las plantas y la respuesta a los factores de producción. Con respecto a la fertilización y al distanciamiento, las recomendaciones para el cultivo de la sandía fertirrigada se basan principalmente en cultivos de secano. Hay escasez de informaciones sobre la densidad poblacional y de la fertirrigación para el cultivo de sandía.

El objetivo de este trabajo fue evaluar el efecto de diferentes dosis de nitrógeno y potasio, aplicadas por fertirrigación, y de la distancia entre plantas sobre la productividad del híbrido Shadow de sandía sin semillas.

\section{Materiales y Métodos}

El experimento fue realizado entre el 5 de agosto y el 31 de octubre de 2008, en el Municipio de Tupã, SP, Brazil, a $21^{\circ} 56^{\prime} 05^{\prime \prime} \mathrm{S}, 50^{\circ} 30^{\prime} 49^{\prime \prime} \mathrm{W}$ y $524 \mathrm{~m}$ de altitud. De acuerdo con la clasificación de Köppen, el clima es tropical, con estación seca en invierno (Aw), y precipitación media anual de $1.290,9 \mathrm{~mm}$ y temperatura de $24,5^{\circ} \mathrm{C}$. Durante el período experimental, la precipitación total fue $97,1 \mathrm{~mm}$; las lluvias empezaron 32 días después del trasplante (DDT), con mayor precipitación $(32,7 \mathrm{~mm})$ a partir de los 57 DDT. El suelo de la zona es un Argissolo Vermelho-Amarelo (Santos et al., 2006), con 15 años de cultivo con pastos. $\mathrm{El}$ análisis químico del suelo presentó: $\mathrm{pH}\left(\mathrm{CaCl}_{2}\right), 4,2$; MO, $9 \mathrm{~g} \mathrm{dm}^{-3} ; \mathrm{K}, 1,5 \mathrm{mmol}_{\mathrm{c}} \mathrm{dm}^{-3} ; \mathrm{Ca}, 8,0 \mathrm{mmol}_{\mathrm{c}} \mathrm{dm}^{-3}$; $\mathrm{Mg}, \quad 5,0 \mathrm{mmol}_{\mathrm{c}} \mathrm{dm}^{-3} ; \mathrm{H}+\mathrm{Al}, \quad 18,0 \mathrm{mmol}_{\mathrm{c}} \mathrm{dm}^{-3}$; SB (suma de bases), 15,0 $\mathrm{mmol}_{\mathrm{c}} \mathrm{dm}^{-3} ;$ y CIC ( $\mathrm{pH}=7), 33,0 \mathrm{mmol}_{\mathrm{c}} \mathrm{dm}^{-3}$; P (resina), 3,0 $\mathrm{mg} \mathrm{dm}^{-3}$; $\mathrm{B}, 0,16 \mathrm{mg} \mathrm{dm}^{-3} ; \mathrm{Zn}, 0,2 \mathrm{mg} \mathrm{dm}^{-3} ; \mathrm{V}, 45 \%$. El calcáreo agrícola, con $120 \%$ de PRNT (poder relativo de neutralización total) fue aplicado, para elevar la saturación de bases a 70\% (Trani et al., 1997).

Se utilizó el diseño experimental de bloques al azar, en parcelas subdivididas, con cuatro tratamientos principales - dosis de $\mathrm{N}+\mathrm{K}_{2} \mathrm{O}$ con $79,8+106,7$, $106,4+142,2,133,0+177,7$, y $159,6+213,2 \mathrm{~kg} \mathrm{ha}^{-1}$, respectivamente $-\mathrm{y}$ cuatro tratamientos secundarios, con distancia entre plantas de $0,5,1,0,1,5$ y $2,0 \mathrm{~m}$, con tres repeticiones. Las dosis de referencia para $\mathrm{N}$ $+\mathrm{K}_{2} \mathrm{O}\left(106,4+142,2 \mathrm{~kg} \mathrm{ha}^{-1}\right)$ fueron exportadas por los frutos con semillas del híbrido Tide, bajo cultivo de secano (Grangeiro \& Cecílio Filho, 2004). Los demás tratamientos correspondieron a 75,125 y $150 \%$ de la dosis recomendada. La distancia entre hileras de plantas fue 2,0 m. En cada unidad experimental, había tres líneas con seis plantas, y se consideró útil la línea central para las evaluaciones.

En la fertilización de plantío por surcos, se aplicó $240 \mathrm{~kg} \mathrm{ha}^{-1}$ de $\mathrm{P}_{2} \mathrm{O}_{5}$ (superfosfato simple), y $40 \mathrm{~kg} \mathrm{ha}^{-1}$ de $\mathrm{MgO}$ (sulfato de magnesio), para aumentar la concentración de $\mathrm{Mg}$ a $9 \mathrm{mmol}_{\mathrm{c}} \mathrm{dm}^{-3}$ (Trani et al., 1997). Las aplicaciones de $1 \mathrm{~kg} \mathrm{ha}^{-1}$ de $\mathrm{B}$ y $3 \mathrm{~kg} \mathrm{ha}^{-1}$ de $\mathrm{Zn}$ fueron realizadas, vía fertirrigación, a los 15 y 30 DDT. Las aplicaciones de $\mathrm{N}+\mathrm{K}$ siguieron las recomendaciones para cada tratamiento.

Se utilizó el sistema de riego por goteo, compuesto por una bomba hidráulica acoplada a un motor eléctrico (2.206 W), filtros de disco, válvulas de retención y de 
regulación, para estandarizar la presión de trabajo en los goteros. Se utilizaron tuberías de PVC para la red primaria $(50 \mathrm{~mm})$, secundaria $(35 \mathrm{~mm})$ y de derivación (35 mm), a las cuales fueron conectadas los tubos con los goteros (1,4 L por hora), distanciados a $0,5 \mathrm{~m}$, con presión del sistema de $100 \mathrm{kPa}$. El agua de riego se obtuvo de un pozo artesiano y mostró la siguiente composición: $35,0,0,75,8,0,4,0,0,1,1,9,0,8,<0,1 \mathrm{y}$ 0,85 $\mathrm{mg} \mathrm{L}^{-1}$ de $\mathrm{CaCO}_{3}, \mathrm{Na}, \mathrm{Ca}, \mathrm{Mg}, \mathrm{B}, \mathrm{Ca}, \mathrm{Cl}, \mathrm{F}, \mathrm{Fe}$, $\mathrm{N}$, respctivamente; $\mathrm{CE}$ de $0,12 \mathrm{dS} \mathrm{m}^{-1} ; \mathrm{pH}$ de $6,36 \mathrm{y}$ RAS de 0,05 .

La aplicación de las dosis $\mathrm{N}+\mathrm{K}_{2} \mathrm{O}$ se hizo en forma individual, con cuatro tanques de polietileno $(1.000 \mathrm{~L})$ y cuatro bombas inyectoras $(552 \mathrm{~W})$, con caudal de hasta $200 \mathrm{~L}$ por hora. Se instalaron filtros de disco e hidrómetros, para controlar la entrada de agua y de nutrientes a inyectar en la tubería del tratamiento específico.

Las fuentes utilizadas de $\mathrm{N}$ y $\mathrm{K}$ fueron nitrato de amonio, nitrato de potasio y nitrato de calcio. Las dosis evaluadas de $\mathrm{NK}_{2} \mathrm{O}$ fueron fraccionadas en 28 fertirrigaciones $\mathrm{y}$, en las primeras nueve, fue aplicado $20 \%$ del total de cada tratamiento. La fertirrigación comenzó 3 DDT y terminó 65 DDT. La aplicación del riego fue realizada mediante la determinación de la evapotranspiración de referencia para el tanque de clase A. Para la conversión del agua evaporada del tanque de clase A (ETCA), en la evapotranspiración de referencia $(\mathrm{ETo})$, se usó la fórmula ETo $=$ ETCA $\times \mathrm{Kp}$, en que el coeficiente de tanque $\mathrm{Kp}$ es igual a 0,7. La evapotranspiración diaria del cultivo (ETc) se determinó con la fórmula ETc $=\mathrm{ETo} \times \mathrm{Kc}$, en que se adaptaron, según Allen et al. (1998), los valores de $\mathrm{Kc}=0,4$ (fase inicial, 0 a $15 \mathrm{DDT}$ ), $\mathrm{Kc}=0,4$ a 1,0 (fase de rápido crecimiento vegetativo, 16 a 32 DDT), $\mathrm{Kc}=1,0$ (fase intermedia, 33 a 75 DDT) y $\mathrm{Kc}=1,0 \mathrm{a}$ 0,75 (fase final, 76 DDT a la cosecha).

Las plántulas del híbrido Shadow (triploide macho estéril), caracterizadas por su vigor, con frutos redondo-ovalados de pulpa roja y peso entre 7 y $8 \mathrm{~kg}$, fueron producidas en el vivero Biotek, en Tupã, SP. El $5 / 8 / 2008$, a los 22 días despues de la siembra, cuando se observó el crecimiento inicial de la segunda hoja, las plántulas fueron trasplantadas. Se utilizó el híbrido Top Gun, en una proporción de 1:3, como polinizador del híbrido Shadow. Fueron instaladas tres colmenas en las márgenes del área experimental, para garantizar la polinización.
El control fitosanitario fue realizado con insecticidas a base de clorhidrato de formetanato y thiamethoxam (trips, pulgón y mosca blanca) y fungicidas a base de difenoconazol, azoxystrobin, thiophanate methyl y chlorothalonil (chancro gomoso del tallo y antracnosis). Las pulverizaciones fueron realizadas tres veces por semana, hasta el inicio de la fructificación, y posteriormente pasó para dos veces por semana, hasta 21 días antes de la cosecha. Después de la instalación de las colmenas, las pulverizaciones con insecticidas fueron realizadas al final de la tarde. Las malezas de hoja angosta fueron controladas con un herbicida a base de haloxifop, y las de hoja ancha en forma manual.

La cosecha fue realizada el $31 / 10 / 2008$, cuando se observó una coloración crema en la parte inferior de los frutos en contacto con el suelo. Las variables evaluadas fueron: concentración foliar de $\mathrm{N}$ y K, en la hoja de diagnóstico del estado nutricional (Trani \& Raij, 1997); número total de frutos (FT); número de frutos comerciales (FC) (mayores de $3 \mathrm{~kg}$ y $\sin$ rajaduras); número de frutos entre 3 y $5 \mathrm{~kg}$ (F35); entre 5 y $7 \mathrm{~kg}$ (F57); entre 7 y $9 \mathrm{~kg}$ (F79); con más de 9 kg (F9); peso promedio de frutos (PF); productividad total (PT); productividad comercial (PC); y productividades entre 3 y $5 \mathrm{~kg}$ (P35), 5 y $7 \mathrm{~kg}$ (P57), 7 y $9 \mathrm{~kg}$ (P79), y con más de $9 \mathrm{~kg}$ (P9). Las evaluaciones fueron realizadas por planta y por área de la subparcela, y los valores se expresaron en quilogramos por hectárea. La productividad fue calculada considerándo-se $7.500 \mathrm{~m}^{2}$ de área efectivamente cultivada, en una hectárea con el híbrido Shadow, una vez que $25 \%$ del área era del híbrido Top Gun, utilizado como polinizador. Con los datos obtenidos se hizo el análisis de variancia. En cuanto a los efectos significativos de los factores dosis de $\mathrm{N}+\mathrm{K}_{2} \mathrm{O}$ y distancia entre plantas, se realizó el análisis de regresión por polinomios ortogonales (SAS Institute, 2000).

\section{Resultados y Discusión}

No se observaron efectos significativos de la interacción entre dosis de $\mathrm{N}+\mathrm{K}_{2} \mathrm{O}$ x distanciamiento $\mathrm{y}$ de los factores en forma individual sobre la concentración foliar de $\mathrm{N}$ (Cuadro 1), y se encontró el valor promedio $\left(29,5 \mathrm{~g} \mathrm{~kg}^{-1}\right)$ en el intervalo adecuado (25 a $50 \mathrm{~g} \mathrm{~kg}^{-1}$ ) para la sandía (Trani \& Raij, 1997). Solamente fue verificado efecto significativo del factor dosis sobre las concentraciones de K en la hoja (27,3 a $35,8 \mathrm{~g} \mathrm{~kg}^{-1}$ ), las cuáles aumentaron linealmente 
$\left(\mathrm{y}=0,1006 \mathrm{x}+20,922 ; \mathrm{R}^{2}=0,83\right)$ al elevar las dosis, y fueron consideradas adecuadas $\left(25 \mathrm{a}_{\left.40 \mathrm{~g} \mathrm{~kg}^{-1}\right) \text { según }}\right.$ Trani \& Raij (1997).

La interacción entre dosis de $\mathrm{N}+\mathrm{K}_{2} \mathrm{O}$ x distancia y el factor $\mathrm{N}+\mathrm{K}_{2} \mathrm{O}$ no tuvieron efectos significativos sobre las características evaluadas, por planta o por hectárea (Cuadro 2). La ausencia de respuesta positiva de las variables al incremento de $\mathrm{N}$ y K podría ser atribuida a la falta de efectos significativos de las dosis de $\mathrm{N}+\mathrm{K}_{2} \mathrm{O}$ en la concentración foliar de $\mathrm{N}$. Aunque significativos para la concentración foliar de $\mathrm{K}$, estos valores estaban dentro del intervalo adecuado para la nutrición de la planta, hecho relevante para no tener una respuesta diferenciada sobre las características evaluadas. Asimismo, la mayor eficiencia de la fertirrigación también contribuyó para disminuir la cantidad de fertilizantes utilizados. Según Papadopoulos (1999), la eficiencia de utilización de $\mathrm{N}$ y K, con riego por goteo, estuvo entre 70 y $80 \%$. La fertirrigación redujo las dosis de $\mathrm{N}$ y $\mathrm{K}$ en $50 \%$ para pimentón (Feitosa Filho et al., 2001) y 20\% en plátano (Teixeira et al., 2007), respecto a la fertilización convencional, manteniéndose la productividad. En el presente trabajo,

Cuadro 1. Análisis de variancia para las concentraciones de $\mathrm{N}$ y K $\left(\mathrm{g} \mathrm{kg}^{-1}\right)$, en la hoja de diagnóstico del estado nutricional, en función de las dosis $\mathrm{N}+\mathrm{K}_{2} \mathrm{O}$ y de la distancia entre plantas de sandía del híbrido Shadow.

\begin{tabular}{lcc}
\hline Fuente de variación & $\mathrm{N}$ & $\mathrm{K}$ \\
\hline Dosis $\mathrm{N}+\mathrm{K}_{2} \mathrm{O}(\mathrm{D})$ & $2,67^{\mathrm{ns}}$ & $5,89^{* *}$ \\
Distancia (E) & $0,97^{\mathrm{ns}}$ & $0,33^{\text {ns }}$ \\
D x E & $2,14^{\text {ns }}$ & $0,89^{\text {ns }}$ \\
\hline $\mathrm{CV}(\%)$ & 5,62 & 15,75 \\
\hline
\end{tabular}

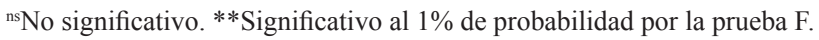

el ahorro de fertilizantes con la fertirrigación podría atribuirse a la capacidad de estimular mayor producción de raíces finas y muy finas en la planta, que generarían efecto positivo en la absorción de nutrientes, lo que concuerda con lo señalado por Coelho et al. (2001).

Las menores dosis evaluadas $\left(79,8+106,7 \mathrm{~kg} \mathrm{ha}^{-1} \mathrm{de}\right.$ $\mathrm{N}+\mathrm{K}_{2} \mathrm{O}$ ), que fueron utilizadas por el hibrido Shadow para crecer y producir, sin diferir de las mayores dosis (Cuadro 2), correspondieron a $25 \%$ menos de las cantidades de nutrientes acumulados en $40 \mathrm{Mg} \mathrm{ha}^{-1}$ de frutos con semillas del híbrido Tide (Grangeiro \& Cecílio Filho, 2004). Estas dosis, consideradas adecuadas para la expresión del potencial productivo del híbrido Shadow, fueron próximas a las reportadas por Andrade Júnior et al. (2006b), para producir $60 \mathrm{Mg} \mathrm{ha}^{-1}$ de la cultivar Crimson Sweet (frutos grandes y con semillas).

La distancia tuvo efectos significativos sobre FT, FC, PT y PC, incluso en las diferentes clases de frutos, en la evaluación por planta (Cuadro 2). El aumento de la distancia entre plantas de 0,5 para $2,0 \mathrm{~m}$ incrementó el FT y FC, incluso en las clases evaluadas, ajustándose significativamente al modelo lineal (Figura 1). Cuando la distancia entre plantas pasó de 0,5 para $2,0 \mathrm{~m}$, hubo aumentos de 100 y 122\% en F35 y F57 que, en conjunto, representaron poco más del $80 \%$ del FC en los distanciamientos evaluados.

Resende \& Costa (2003), Miranda et al. (2005) y Walters (2009) también observaron una reducción en el número de frutos por planta, cuando se aumentó la densidad. Según Motsenbocker \& Arancibia (2002), una cultivar con frutos de mayor peso mostró mayor variación en el número de frutos por planta, y un incremento cuando se aumentó la densidad, mientras

Cuadro 2. Análisis de variancia para el número y productividad de frutos total (FT, PT), comercial (FC, PC), de frutos con 3 a $5 \mathrm{~kg}$ (F35, P35), con 5 a $7 \mathrm{~kg}$ (F57, P57), con 7 a $9 \mathrm{~kg}$ (F79, P79) y con más de $9 \mathrm{~kg}$ (F9, P9), y peso medio de frutos (PF), por planta y por hectárea, en función de las dosis $\mathrm{N}+\mathrm{K}_{2} \mathrm{O}$ y de la distancia entre plantas de sandía del híbrido Shadow.

\begin{tabular}{|c|c|c|c|c|c|c|c|c|c|c|c|c|c|c|}
\hline Fuente de variación & FT & $\mathrm{FC}$ & F35 & F57 & F79 & F9 & $\mathrm{PF}$ & PT & $\mathrm{PC}$ & P35 & P57 & P79 & P9 & $\mathrm{PF}$ \\
\hline & \multicolumn{14}{|c|}{ Por planta } \\
\hline Dosis $\mathrm{N}+\mathrm{K}_{2} \mathrm{O}(\mathrm{D})$ & $0,18^{\mathrm{ns}}$ & $0,17^{\mathrm{ns}}$ & $0,77^{\mathrm{ns}}$ & $0,70^{\text {ns }}$ & $0,51^{\mathrm{ns}}$ & $0,71^{\mathrm{ns}}$ & - & $0,06^{\mathrm{ns}}$ & $0,04^{\mathrm{ns}}$ & $0,68^{\mathrm{ns}}$ & $0,37^{\mathrm{ns}}$ & $0,55^{\text {ns }}$ & $0,69^{\text {ns }}$ & $0,74^{\mathrm{ns}}$ \\
\hline Distancia $(\mathrm{E})$ & $32,44^{* *}$ & $30,14^{* *}$ & $8,97 * *$ & $16,53 * *$ & $18,64 * *$ & $7,61 * *$ & - & $35,01 * *$ & $32,78 * *$ & $4,78 * *$ & $17,71 * *$ & $19,01 * *$ & $6,15^{* *}$ & $1,04^{\mathrm{ns}}$ \\
\hline$\underline{\mathrm{D} \times \mathrm{E}}$ & $0,25^{\mathrm{ns}}$ & $0,12^{\mathrm{ns}}$ & $0,77^{\mathrm{ns}}$ & $0,84^{\mathrm{ns}}$ & $1,41^{\mathrm{ns}}$ & $1,67^{\mathrm{ns}}$ & - & $0,35^{\mathrm{ns}}$ & $0,29^{\text {ns }}$ & $0,60^{\text {ns }}$ & $0,87^{\mathrm{ns}}$ & $1,53^{\mathrm{ns}}$ & $1,69^{\mathrm{ns}}$ & $0,83^{\mathrm{ns}}$ \\
\hline \multirow[t]{2}{*}{ CV (\%) } & 20,55 & 22,02 & 34,08 & 28,07 & 41,32 & 78,29 & - & 21,17 & 22,26 & 41,50 & 28,78 & 41,42 & 82,12 & 8,35 \\
\hline & \multicolumn{14}{|c|}{ Por hectárea } \\
\hline Dosis $\mathrm{N}+\mathrm{K}_{2} \mathrm{O}$ (D) & $0,06^{\mathrm{ns}}$ & $0,10^{\mathrm{ns}}$ & $0,55^{\mathrm{ns}}$ & $0,79^{\mathrm{ns}}$ & $0,05^{\text {ns }}$ & $1,36^{\mathrm{ns}}$ & - & $0,06^{\mathrm{ns}}$ & $0,10^{\mathrm{ns}}$ & $0,55^{\mathrm{ns}}$ & $0,79^{\text {ns }}$ & $0,05^{\text {ns }}$ & $1,36^{\mathrm{ns}}$ & - \\
\hline Distancia (E) & $17,39 * *$ & $15,05^{* *}$ & $5,44 * *$ & $9,04 * *$ & $0,60^{\mathrm{ns}}$ & $0,45^{\mathrm{ns}}$ & - & $17,39 * *$ & $15,05^{* *}$ & $5,44 * *$ & $9,04 * *$ & $0,60^{\mathrm{ns}}$ & $0,45^{\mathrm{ns}}$ & - \\
\hline$\underline{\mathrm{D}} \mathrm{xE}$ & $0,48^{\mathrm{ns}}$ & $0,20^{\mathrm{ns}}$ & $0,31^{\mathrm{ns}}$ & $1,18^{\mathrm{ns}}$ & $1,47^{\mathrm{ns}}$ & $1,13^{\text {ns }}$ & - & $0,48^{\mathrm{ns}}$ & $0,20^{\mathrm{ns}}$ & $0,31^{\mathrm{ns}}$ & $1,18^{\mathrm{ns}}$ & $1,47^{\mathrm{ns}}$ & $1,13^{\text {ns }}$ & - \\
\hline$\overline{\mathrm{CV}(\%)}$ & 21,24 & 20,81 & 46,10 & 30,37 & 40,92 & 93,33 & - & 21,24 & 20,81 & 46,10 & 30,37 & 40,92 & 93,33 & - \\
\hline
\end{tabular}

${ }^{\mathrm{n}}$ No significativo. **Significativo al $1 \%$ de probabilidad por la prueba $\mathrm{F}$. 
que para una cultivar con frutos de menor peso, el incremento fue menor. Sin embargo, Ramos et al. (2009) no observaron aumento en el número de frutos por planta, cuando aumentaron la distancia en cultivares de frutos pequeños.

Las productividades observadas aumentaron linealmente con mayores distancias, en razón del aumento de frutos por planta (Figura 1). La menor densidad de plantas $(2,0 \times 2,0 \mathrm{~m})$ proporcionó incremento de $150 \%$ en la $\mathrm{PC}$, principalmente en P57. De acuerdo con los resultados, con aumento de la densidad, hubo tendencia de reducción del número de frutos por planta, debido a una mayor competición, lo que afectó el crecimiento. Esta respuesta fisiológica también fue observada por Nesmith (1993), Duthie et al. (1999) y Motsenbocker \& Arancibia (2002).
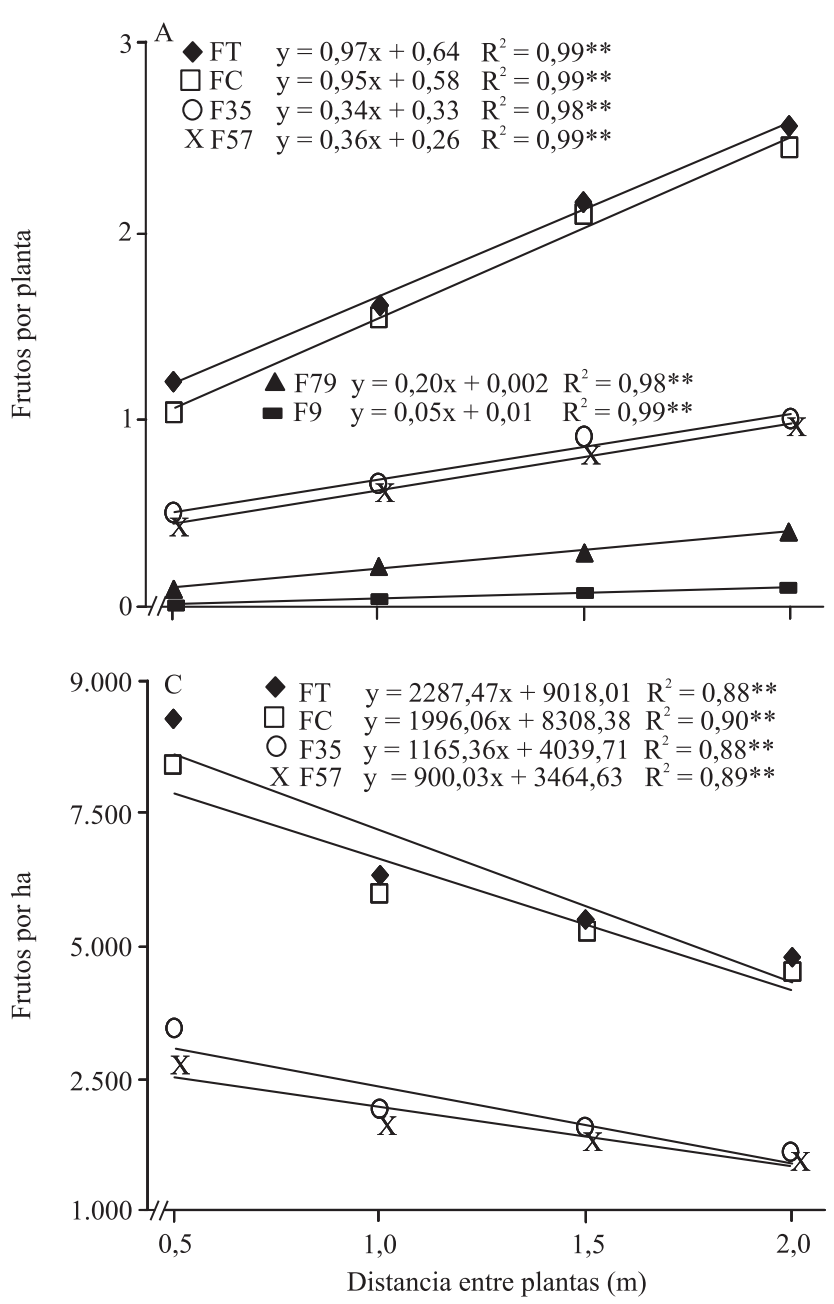

El aumento en la distancia entre plantas disminuyó el número de frutos no comerciales. Con distancia de 0,5 m entre plantas, el número de frutos no comerciales correspondió a $12 \%$ del FT, una disminución de sólo $4 \%$ sobre el mayor distanciamiento evaluado $(2,0$ $\mathrm{m})$. En consecuencia, la PC por planta, en las mismas condiciones, aumentó de 96 a 98\% de la PT. Resende \& Costa (2003) y Miranda et al. (2005) también indicaron que menores distanciamientos entre plantas resultaron en mayor porcentaje de frutos no comerciales.

El PF no fue afectado significativamente por la interacción o por los factores en forma individual. Este resultado difirió de los de Sanders et al. (1999), García \& Souza (2002) y Miranda et al. (2005), quienes observaron que la reducción en el distanciamiento disminuyó el peso de los frutos. Pero en el presente estudio, la disminución
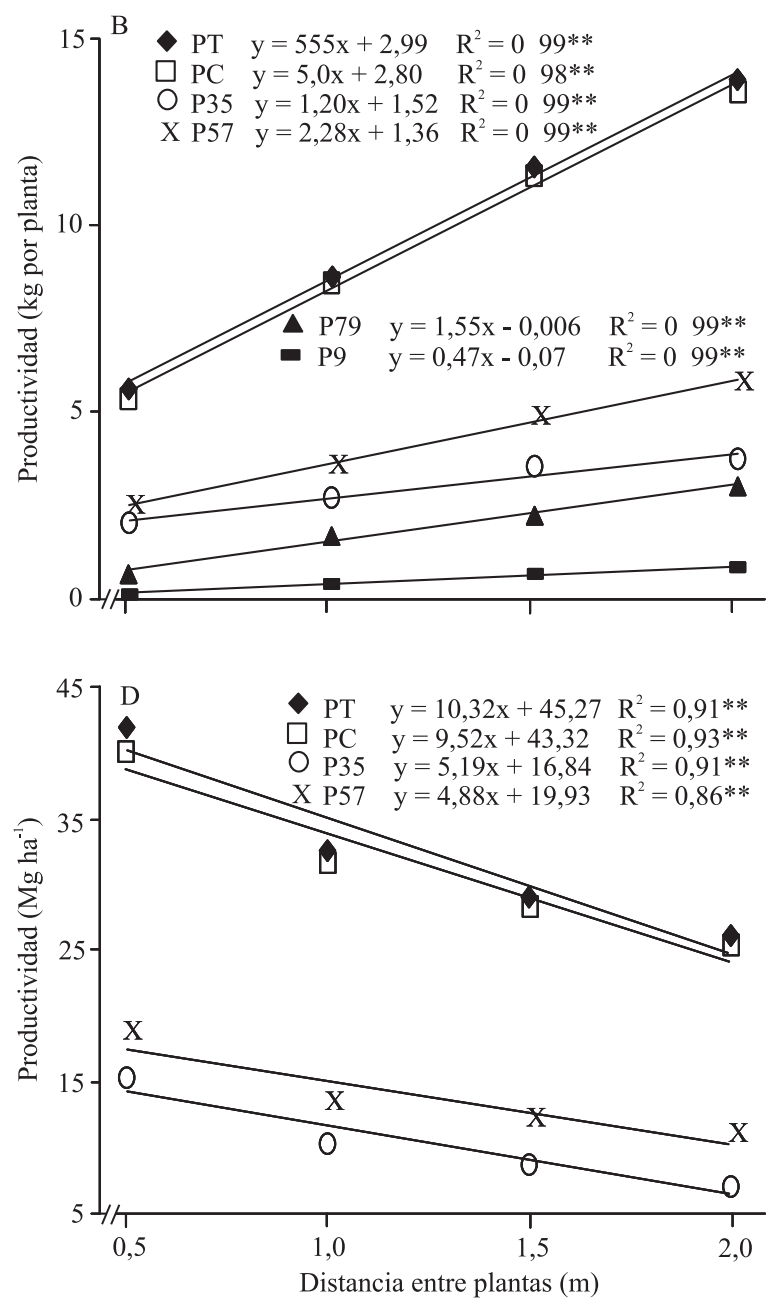

Figura 1. Número de frutos (A, C) y productividad (B, D) total (FT, PT), comercial (FC, PC), y de frutos con 3 a $5 \mathrm{~kg}$ (F35, P35), con 5 a $7 \mathrm{~kg}$ (F57, P57), con 7 a $9 \mathrm{~kg}$ (F79, P79) y con más de $9 \mathrm{~kg}$ (F9, P9), en función de la distancia entre plantas de sandía del híbrido Shadow. 
del distanciamiento de 2,0 para $0,5 \mathrm{~m}$, no resultó en menor peso de frutos (promedio igual a $5,46 \mathrm{~kg}$ por fruto). La divergencia entre los resultados se podría deber al grupo al que pertenecen las cultivares utilizadas. En el presente experimento, se evaluó el híbrido Shadow, de frutos pequeños ( 7 a $8 \mathrm{~kg}$ ), mientras que los autores mencionados utilizaron cultivares con mayor tamaño de fruto, que respondieron más a la variación sobre el distanciamiento entre plantas.

Hubo efecto individual del factor distancia solamente para las características FT, FC, F35, F57, PT, PC, P35 y P57, por hectárea (Cuadro 2), que se ajustaron a ecuaciones lineales (Figura 1) y describieron respuestas en sentido inverso, cuando fueron evaluadas y expresadas por planta. Es decir, el aumento del distanciamiento entre las plantas causó una reducción en el FT y PT; FC y PC; F35 y P35; F57 y P57 por hectárea.

Estos resultados coinciden con los reportados por Nesmith (1993), Motsenbocker \& Arancibia (2002) y Walters (2009), quienes observaron un mayor número de frutos por hectárea y una mayor producción en densidades más altas. No obstante, Resende \& Costa (2003) obtuvieron mayores productividades con la menor densidad, pero con una mayor densidad aumentó el porcentaje de frutos no comerciales. Asimismo, Miranda et al. (2005) no observaron diferencias significativas en la productividad comercial y en el número de frutos por hectárea, al usar distancias de $2,0 \times 1,0 \mathrm{~m}$ (una y dos plantas por hoyo) y de $2,0 \times 1,5 \mathrm{~m}$ (dos plantas por hoyo).

El efecto favorable de una mayor distancia sobre la planta, con aumentos de 116 y $140 \%$ en FT y FC, no fue suficiente para compensar el menor número de plantas por hectárea. En consecuencia, incluso con un aumento significativo de frutos por planta en la mayor distancia (menor densidad poblacional correspondiente a 2.500 plantas ha ${ }^{-1}$ ), los valores para FT, FC, F35 y F57 fueron menores. Por ejemplo, cuando se optó por la distancia de $2,0 \mathrm{~m}$ en lugar de $0,5 \mathrm{~m}$ entre plantas, se observó una reducción de $40 \%$ en el FC, lo que equivalió a menos 2.994 frutos por hectárea.

El mayor aumento en el FT por hectárea, al reducir la distancia entre plantas, provocó un aumento proporcional de la PT. La mayor PC $\left(38,56 \mathrm{Mg} \mathrm{ha}^{-1}\right)$, obtenida con la distancia de $0,5 \mathrm{~m}$ entre plantas, fue cerca de 58,8\% superior a la PC con la distancia de 2,0 m; sin embargo, la PC por planta fue mayor en $150 \%$ al usar la distancia de $2,0 \mathrm{~m}$, en comparación a $0,5 \mathrm{~m}$.
La mayor PC fue compuesta por $14,24 \mathrm{Mg} \mathrm{ha}^{-1}$ en P35 y $17,49 \mathrm{Mg} \mathrm{ha}^{-1}$ en P57, que correspondieron a 36,9 y $45,3 \%$ de la PC y, juntos, sumaron $82,2 \%$. Este resultado fue importante, pues las tendencias más recientes en los mercados internos y externos de sandía indican una preferencia creciente por frutos con menor peso, inferiores a $6 \mathrm{~kg}$ (Resende \& Costa, 2003). Con respecto a las características P79 y P9 por hectárea, que no fueron influenciadas significativamente por la distancia, la productividad promedio en estas clases fue de 5,12 y $1,14 \mathrm{Mg} \mathrm{ha}^{-1}$, cerca de 13,3 y $3,0 \%$ de la PC.

Walters (2009) también observó que el número de frutos, en sandía con menos de $1,4 \mathrm{~kg}$, aumentó de $3,3 \%$, en la menor densidad, a $10,8 \%$ en la densidad más alta, con incremento en la productividad de 621 a $3.903 \mathrm{~kg} \mathrm{ha}^{-1}$. Este autor resaltó que, en la mayor densidad, cerca de $80 \%$ de la producción y de número de frutos se concentraron en sandías de 1,4 a $3,7 \mathrm{~kg} \mathrm{y}$, en la menor densidad, se redujo a $50 \%$; además, la productividad y el número de frutos en sandías de 3,7 a 5,9 kg disminuyó con el aumento de la densidad.

\section{Conclusiones}

1. El aumento de las dosis de $\mathrm{N}+\mathrm{K}_{2} \mathrm{O}$ no incrementa la productividad de la sandía híbrido Shadow, independientemente de la población de plantas.

2. La productividad total y comercial de la sandía híbrido Shadow, disminuye linealmente con el aumento de la distancia entre plantas.

3. En las condiciones experimentales descritas para el híbrido de sandía Shadow cultivado con fertirrigación y con 2,0 m entre hileras, es adecuado el distanciamiento de $0,5 \mathrm{~m}$ entre plantas y las dosis de $79,8 \mathrm{~kg} \mathrm{ha}^{-1}$ de $\mathrm{N}$ y $106,7 \mathrm{~kg} \mathrm{ha}^{-1}$ de $\mathrm{K}_{2} \mathrm{O}$.

\section{Agradecimientos}

A la Fundação de Amparo à Pesquisa do Estado de São Paulo, por la financiación del proyecto; y a los propietarios del vivero de plantones Biotek, por la cesión de las instalaciones y por el apoyo tecnológico.

\section{Referencias}

ALFAIA, S.S. Destino de fertilizantes nitrogenados $\left({ }^{15} \mathrm{~N}\right)$ em um Latossolo Amarelo cultivado com feijão caupi (Vigna unguiculata L.). Acta Amazonica, v.27, p.65-72, 1997. 
ALLEN, R.G.; PEREIRA, L.S.; RAES, D.; SMITH, M. Crop evapotranspiration: guidelines for computing crop water requirements. Roma: FAO, 1998. 300p. (FAO. Irrigation and drainage paper, 56).

ANDRADE JÚNIOR, A.S. de; DIAS, N. da S.; FIGUEIREDO JÚNIOR, L.G.M; RIBEIRO, V.Q.; SAMPAIO, D.B. Produção e qualidade de frutos de melancia à aplicação de nitrogênio via fertirrigação. Revista Brasileira de Engenharia Agrícola e Ambiental, v.10, p.836-841, 2006a.

ANDRADE JÚNIOR, A.S. de; DIAS, N. da S.; RODRIGUES, B.H.N.; MELO, F. de B.; RIBEIRO, V.Q. Fertirrigação nitrogenada e potássica na cultura da melancia. Teresina: Embrapa Meio-Norte, 2006b. 6p. (Embrapa Meio-Norte. Comunicado técnico, 182).

COELHO, E.F.; OLIVEIRA, F. das C.; ARAÚJO, E.C.E.; VASCONCELOS, L.F.L.; LIMA, D.M. Distribuição do sistema radicular da mangueira sob irrigação localizada em solo arenoso de Tabuleiros Costeiros. Revista Brasileira de Fruticultura, v.23, p.250-256, 2001.

DUTHIE, J.A.; SCHREFLER, J.W.; ROBERTS, B.W.; EDELSON, J.V. Plant density-dependent variation in marketable yield, fruit biomass, and marketable fraction in watermelon. Crop Science, v.39, p.406-412, 1999.

FEITOSA FILHO, J.C.; LEITE JÚNIOR, G.P.; CAVALCANTE, L.F.; LOPES, W.F.; SANTOS, C.S.; LOPES, W.F; PINTO, J.M. Resposta da cultura do pimentão a diferentes doses de $\mathrm{N}$ e $\mathrm{K}$ aplicadas por fertirrigação em comparação à adubação convencional. In: WORKSHOP FERTIRRIGAÇÃO, FLORES, FRUTAS E HORTALIÇAS, 2., 2001, Piracicaba. Anais. Piracicaba: ESALQ, 2001. p.19-31.

FILGUEIRA, F.A.R. Novo manual de olericultura: agrotecnologia moderna na produção e comercialização de hortaliças. Viçosa: UFV, 2008. 421p.

GARCÍA, L.F.; SOUZA, V.A.B. de. Influência do espaçamento e da adubação nitrogenada sobre a produção da melancia. Revista de la Facultad de Agronomía, v.28, p.59-70, 2002.

GRANGEIRO, L.C.; CECÍLIO FILHO,A.B.Acúmulo e exportação de macronutrientes em melancia sem sementes. Horticultura Brasileira, v.23, p.763-767, 2005.

GRANGEIRO, L.C.; CECÍLIO FILHO,A.B.Acúmulo e exportação de macronutrientes pelo híbrido de melancia Tide. Horticultura Brasileira, v.22, p.93-97, 2004.

INSTITUTO FNP. Agrianual 2009: anuário da agricultura brasileira. São Paulo: FNP, 2009. 497p.

MIRANDA, F.R. de; MONTENEGRO, A.A.T.; OLIVEIRA, J.J.G. Produtividade da melancia irrigada por gotejamento em diferentes espaçamentos de plantio. Revista Ciência Agronômica, v.36, p.158-162, 2005.

MORAIS, N.B.; BEZERRA, F.M.L.; MEDEIROS, J.F.; CHAVES, S.W.P. Resposta de plantas de melancia cultivadas sob diferentes níveis de água e de nitrogênio. Revista Ciência Agronômica, v.39. p.369-377,2008.
MOTSENBOCKER, C.E.; ARANCIBIA, R.A. In-row spacing influences triploid watermelon yield and crop value. HortTechnology, v.12, p.437-440, 2002.

NESMITH, D.S. Plant spacing influences watermelon yield and yield components. HortScience, v.28, p.885-887, 1993.

PAPADOPOULOS, I. Fertigation: present situation and future prospects. In: FOLEGATTI, M.V. (Ed.). Fertirrigação: citrus, flores, hortaliças. Guaíba: Agropecuária, 1999. p.85-154.

RAMOS, A.R.P.; DIAS, R. de C.S.; ARAGÃO, C.A. Avaliação de produtividade de melancia de frutos pequenos no Submédio do São Francisco, em função da densidade de plantio. Horticultura Brasileira, v.27, p.S2189-S2195, 2009.

RESENDE, G.M. de; COSTA, N.D. Características produtivas da melancia em diferentes espaçamentos de plantio. Horticultura Brasileira, v.21, p.695-698, 2003.

ROBINSON, R.W.; WALTERS, D.S. Cucurbits. New York: CAB International, 1997. 226p.

SANDERS, D.C.; CURE，J.D.; SCHULTHEIS，J.R. Yield response of watermelon to planting density, planting pattern, and polyethylene mulch. HortScience, v.34, p.1221-1223, 1999.

SANTOS, H.G. dos; JACOMINE, P.K.T.; ANJOS, L.H.C. dos; OLIVEIRA, V.A. de; OLIVEIRA, J.B. de; COELHO, M.R.; LUMBRERAS, J.F.; CUNHA, T.J.F. (Ed.). Sistema brasileiro de classificação de solos. 2.ed. Rio de Janeiro: Embrapa Solos, 2006. $306 \mathrm{p}$.

SAS INSTITUTE. SAS procedures guide for computers. Version 8.1. Cary: SAS Institute, 2000. 1686p.

TEIXEIRA, L.A.J.; NATALE, W.; MARTINS, A.L.M. Nitrogênio e potássio via fertirrigação e adubação convencional: estado nutricional das bananeiras e produção de frutos. Revista Brasileira de Fruticultura, v.29, p.153-160, 2007.

TRANI, P.E.; PASSOS, F.A.; NAGAI, H.; MELO, A.M.T. Melão e melancia. In: RAIJ, B. van; CANTARELLA, H.; QUAGGIO, J.S.; FURLANI, A.M.C. (Ed.). Recomendações de adubação e calagem para o Estado de São Paulo. Campinas: Instituto Agronômico, 1997. p.181 (IAC. Boletim técnico, 100).

TRANI, P.E.; RAIJ, B. van. Hortaliças. In: RAIJ, B. van; CANTARELLA, H.; QUAGGIO, J.S.; FURLANI, A.M.C. (Ed.). Recomendações de adubação e calagem para o estado de São Paulo. Campinas: Instituto Agronômico, 1997. p.157-164. (IAC. Boletim técnico, 100).

VILLAS BÔAS, R.L.; ANTUNES, C.L.; BOARETO, A.E.; SOUSA, V.F.; DUENHAS, L.H. Perfil da pesquisa e emprego da fertirrigação no Brasil. In: FOLEGATTI, M.V.; CASARINI, E.; BLANCO, F.F.; BRASIL, R.P.C. do; RESENDE, R.S. (Ed.). Fertirrigação: flores, frutas e hortaliças. Guaíba: Agropecuária, 2001. p.71-103.

WALTERS, S.A. Influence of plant density and cultivar on mini triploid watermelon yield and fruit quality. HortTechnology, v.19, p.553-557, 2009.

Recibido el 12 abril de 2011 y aceptado el 2 agosto de 2011 